\title{
Improved Equation for Shear Modulus Using Four-Point Ring-Twist Test Data
}

\author{
C. W. BERT ${ }^{1}$ \\ School of Aerospace and Mechanical Engineering, The University of \\ Oklahoma, Norman, OK 73019-1052
}

\begin{abstract}
There are numerous test-specimen configurations for determining the static shear modulus of composites. Selection of specimen configuration depends primarily on product component configuration. Two-ring specimens in use are the split-ring twist specimen and the four-point-loading ring-twist specimen. The latter is more advantageous, since it uses an intact ring. However, reported validation tests on aluminum rings showed a large error in predicted shear modulus. The present paper provides an improved analysis that takes into account the tilting of the cross section on the loading bar.
\end{abstract}

\section{INTRODUCTION}

NINCE FIBER-REINFORCED COMPOSITES have considerably lower shear $\checkmark$ moduli/elastic moduli ratios than homogeneous materials, shear deformation is more important in composite structures. Consequently, a great variety of specimen configurations has been proposed and used for determination of shear moduli of composites (Tarnopol'skii and Kincis, 1984).

The crux of the selection of an appropriate shear test is the geometric configuration of material available for the specimen. Thus, if the specimen material is in the form of a thin laminate, the following tests may be used: panel shear or picture frame (Penton, 1960), plate twist (Whitney, 1967), rail shear (Whitney et al., 1971), \pm 45 -degree tension, and off-axis tension (Chamis and Sinclair, 1977), cruciform sandwich beam (Jones, 1975), and Iosepescu (for shear strength only). If the specimen configuration is in the form of a bar, the three-point and five-point beam tests (Pfafrod et al., 1972) and the torsion bar test may be used. A popular product configuration, especially for filament-wound material, is a long tube, for which the torsion tube test (Whitney, 1967) can be used. However, in many instances, there is insufficient axial length of material available for the torsion tube test. For this case, ring tests are appropriate. The split-ring test, sometimes called the Douglas split-ring test, was introduced by Greszczuk (1968). Later, to avoid

$\overline{{ }^{1} \text { E-mail: cbert@ou.edu }}$

Journal of Thermoplastic COMPOSite MAterials, Vol. 14-November 2001

489

0892-7057/01/06 0489-09 \$10.00/0 DOI: 10.1106/GNG0-LR9B-FFKB-RV13

(C) 2001 Sage Publications 


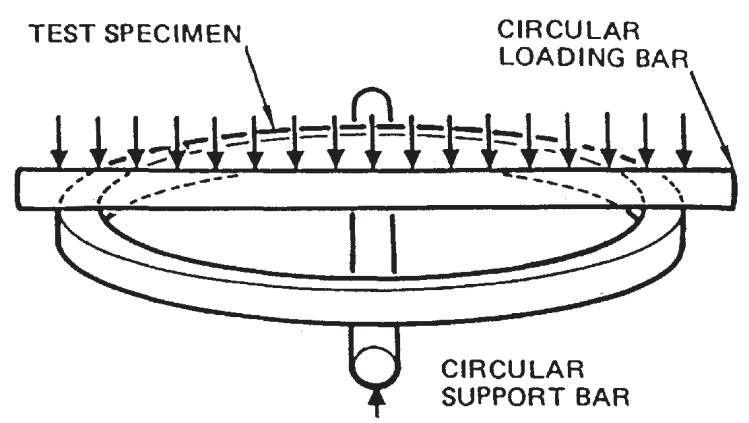

Figure 1. Schematic diagram of the four-point-load test setup (from Greszczuk, 1979).

the need for actually forming a split, Kintsis and Shlitsa (1978) and Greszczuk $(1979,1981)$ introduced the four-point-load ring test (see Figure 1).

To validate the four-point-load ring setup and data-reduction equations, Greszczuk (1979) used a 6061-T6 aluminum-alloy ring. The result was a predicted shear modulus about $9.3 \%$ higher than the well-established value. The purpose of the present analysis is to develop an improved data-reduction equation taking into account these additional effects, which were neglected in the equation presented by Greszczuk (1979):

! cross-sectional tilting action that moves the effective radius of support inward from the center of the cross section

! out-of-plane transverse shear deformation

ANALYSIS
A schematic diagram of the test setup used by Greszczuk is shown in Figure 1
Now consider one half of the plan view as shown in Figure 2 . Here $P$ represents the
total applied load; thus, in Figure 1 each load point carries a load of $P / 2$. However
in Figure 2 , due to the "cuts" at points $A$ and $C$, these points carry only half of the
load at these points, namely, $P / 4$. Also, due to the cuts, resisting bending moments,
$M_{A}$ and $M_{c}$, must be placed at points $A$ and $C$, respectively. It is noted that the load
points are neither at the centroid of the cross section nor at the inner edge of the
cross section, but are at an "effective" radius $R_{e}$, due to the loading not being dis-
tributed uniformly across the whole cross section of the ring.
Taking moments about line $A C$ and noting that $M_{C}=M_{A}$ due to symmetry gives
\[ M_{A}=P R_{e} / 4 \]

Now consider the equilibrium of the cross section located at a circumferential 


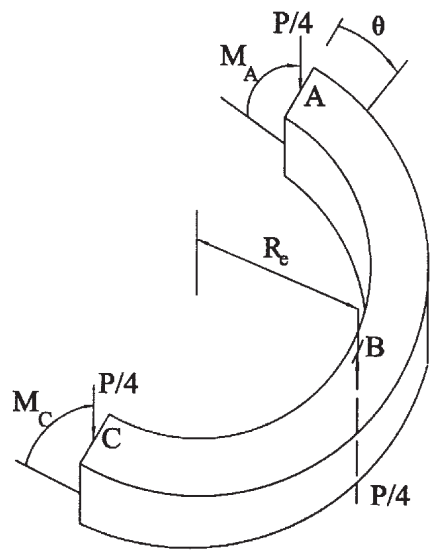

Figure 2. Loading of one half of the ring.

position angle $\theta$, measured from cross section $A$ (see Figure 3 ). The bending moment, torque, and transverse shear at the center of the cross section at $\theta$ are given by

$$
\begin{gathered}
M(\theta)=M_{A} \cos \theta-\left(P R_{e} / 4\right) \sin \theta \\
T(\theta)=M_{A} \sin \theta-(P / 4)\left(R-R_{e} \cos \theta\right) \\
V(\theta)=P / 4
\end{gathered}
$$

where $R$ is the mean radius of the ring.

The total strain energy in one quarter of the $\operatorname{ring}(\operatorname{arc} A B)$ is the sum of those due to bending, twisting, and transverse shear:

$$
U_{A B}=\int_{0}^{\pi / 2}\left(\frac{M^{2} R}{2 E_{\theta} I}+\frac{T^{2} R}{2 G_{\theta z} J}+\frac{V^{2} R}{2 K G_{\theta z} A}\right) d \theta
$$

where $A=$ cross-sectional area of the ring, $E_{\theta}=$ circumferential elastic modulus, $G_{\theta z}=$ shear modulus associated with shear stress $\tau_{\theta z}$ (see Figure 4), $I=$ rectangular area moment of inertia of the cross section about its neutral axis for out-of-plane bending, $J=$ torsional factor for the cross section, and $K=$ transverse shear correction factor.

Now for a rectangular cross section

$$
J=\beta a b^{3}, K \approx 5 / 6
$$




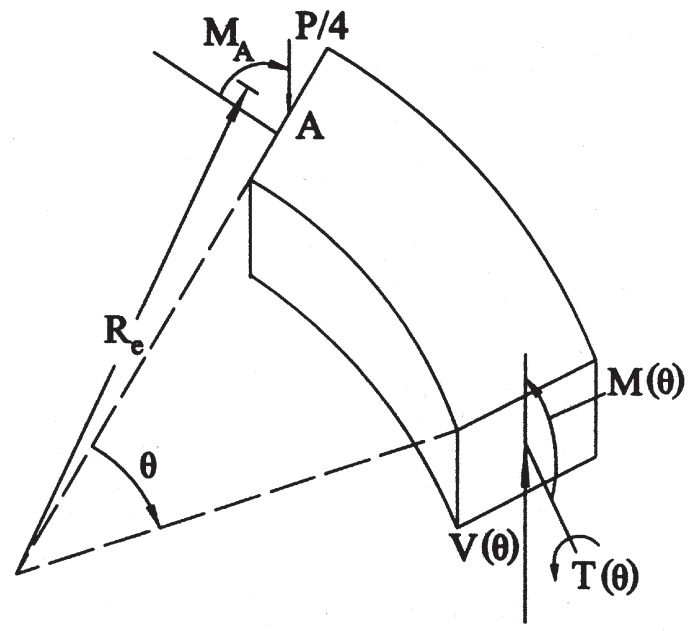

(a)

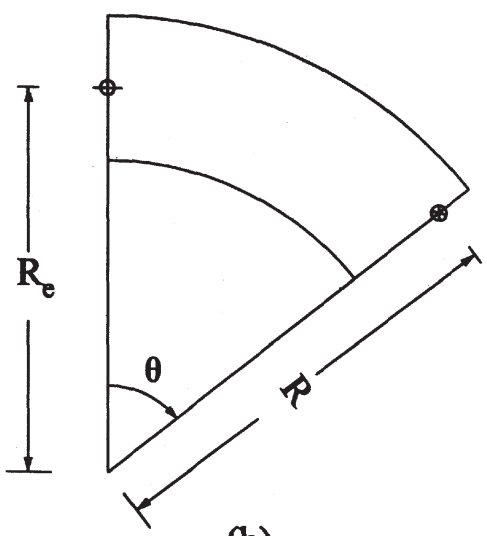

(b)

Figure 3. Free-body diagram: (a) schematic and (b) plan view. 


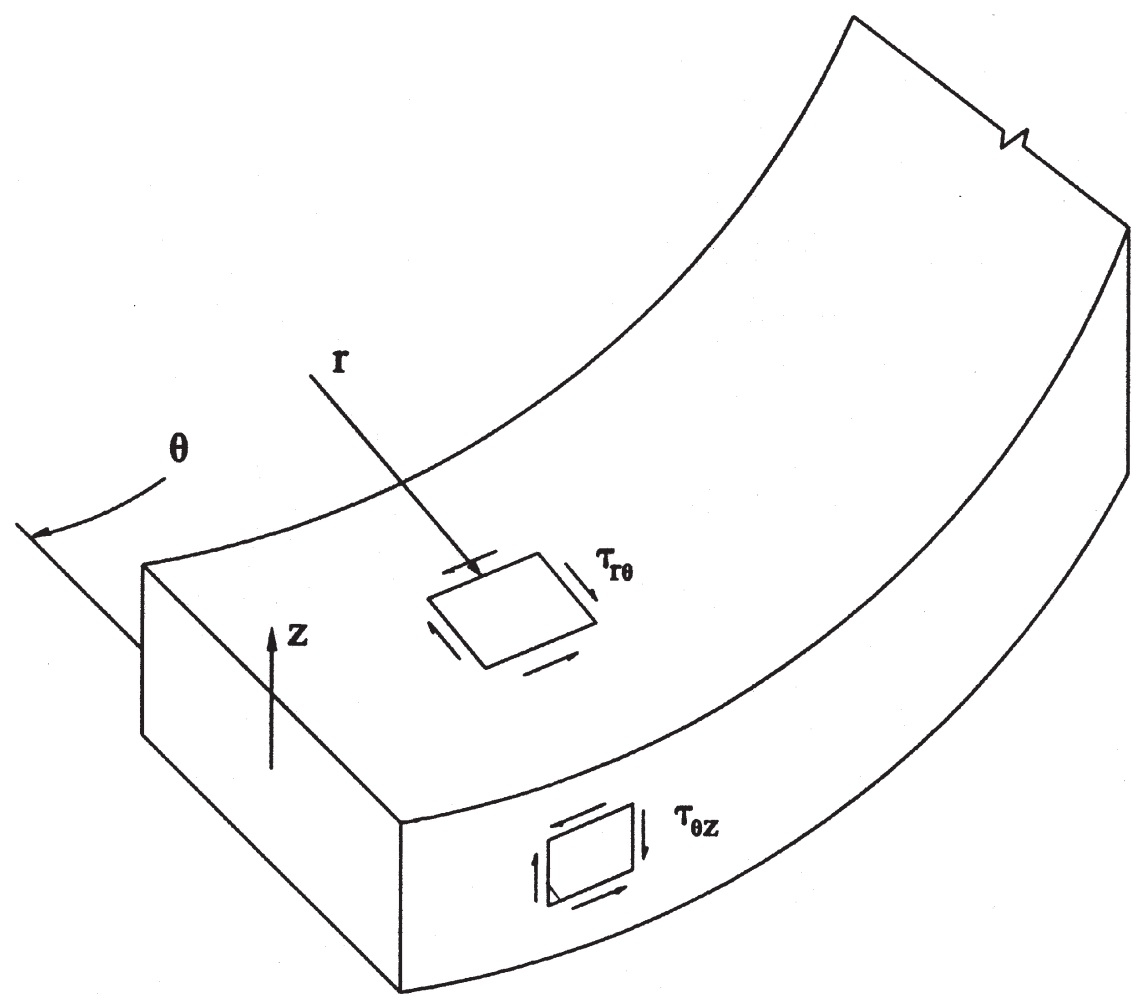

Figure 4. Shear stress components.

where $a=$ axial depth of the cross section, $b=$ radial width of the cross section and $\beta=$ dimensionless coefficient that depends upon the cross-sectional geometry and the orthotropic shear moduli via the ratio $d$ defined as

$$
d=(b / a)\left(G_{r \theta} / G_{\theta z}\right)^{1 / 2}
$$

where $G_{r \theta}$ is the orthotropic shear modulus associated with the shear stress $\tau_{\mathrm{r} \theta}$ (see Figure 4). It is noted that the roles of $a$ and $b$ (and $G_{r \theta}$ and $G_{\theta z}$ ) are the reverse of those assigned by Lekhnitskii (1981).

According to Castigliano's theorem, the out-of-plane deflection $\delta$ is given by

$$
\delta=\partial U_{A B} / \partial(P / 4)
$$

Substituting Equations (1)-(4) into Equation (5) and performing the necessary integration in Equation (8) yields 


$$
\delta=\frac{\pi-2}{8} \frac{P R R_{e}^{2}}{E_{\theta} I}+\left[\left(\frac{\pi}{2}+1\right) R_{e}^{2}-4 R R_{e}+\frac{\pi}{2} R^{2}\right] \frac{P R}{4 G_{\theta z} J}+\frac{\pi}{8} \frac{P R}{K G_{\theta z} A}
$$

For the simplified case considered by Greszczuk (1979), $R_{e} \rightarrow R$ and $1 / K \rightarrow 0$. Then

$$
\delta=\frac{\pi-2}{8} \frac{P R^{3}}{E_{\theta} I}+\frac{\pi-3}{4} \frac{P R^{3}}{G_{\theta z} J}
$$

Solving for $G_{\theta z}$ in Equation (9), one obtains

$$
G_{\theta z}=\frac{\left[\left(\frac{\pi}{2}+1\right) R_{e}^{2}-4 R R_{e}+\frac{\pi}{2} R^{2}\right](1 / J)+(\pi / 2 K A)}{(4 / R)(\delta / P)-\left(\frac{\pi}{2}-1\right)\left(R_{e}^{2} / E_{\theta} I\right)}
$$

Knowing the dimensions of the ring, the elastic modulus $E_{\theta}$, and measuring $\delta / P$, one can use Equation (11) to predict the shear modulus. The elastic modulus could be determined by strain or deflection measurements in a diametral tension (or compression) test.

\section{VALIDATION FOR ISOTROPIC MATERIAL}

The data for Greszczuk's 6061-T4 aluminum-alloy ring are listed below:

$$
\begin{gathered}
a=1.08 \mathrm{~cm}(0.425 \mathrm{in}) \\
b=0.582 \mathrm{~cm}(0.229 \mathrm{in}) \\
R=8.113 \mathrm{~cm}(3.194 \mathrm{in}) \\
E=69.0 \mathrm{GPa}\left(10.0 \times 10^{6} \mathrm{psi}\right)
\end{gathered}
$$

From these data, the following additional parameters are determined:

$$
\begin{gathered}
A=a b=0.628 \mathrm{~cm}^{2}\left(0.0973 \mathrm{in}^{2}\right) \\
I=b a^{3} / 12=0.0608 \mathrm{~cm}^{4}\left(0.00146 \mathrm{in}^{4}\right) \\
\beta=0.221 \text { for } a / b=1.86 \text { and } G_{r \theta} / G_{\theta z}=1
\end{gathered}
$$


Table 1. Predicted values of the shear modulus of aluminum ring.

\begin{tabular}{lccc}
\hline & & \multicolumn{2}{c}{ G, GPa (millions of psi) } \\
\cline { 3 - 4 } & & $\begin{array}{c}\text { Without } \\
\text { Transverse }\end{array}$ & $\begin{array}{c}\text { With } \\
\text { Transverse } \\
\text { Loading Case }\end{array}$ \\
\hline Corner contact & $\boldsymbol{R}_{\mathbf{e}}$ & Shear & Shear \\
\hline Triangular contact distribution & $7.823(3.080)$ & $19.2(2.78)$ & $19.6(2.84)$ \\
Parabolic distribution & $8.016(3.156)$ & $25.0(3.63)$ & $25.4(3.69)$ \\
Uniform contact & $8.057(3.172)$ & $26.3(3.81)$ & $26.7(3.87)$ \\
\hline
\end{tabular}

$$
J=0.0470 \mathrm{~cm}^{4}\left(0.00113 \mathrm{in}^{4}\right)
$$

Unfortunately, Greszczuk (1979) did not give the value of $\delta / P$ in his test. However, he gave the value of $G$ obtained by his formula, Equation (10) above: 28.3 $\mathrm{GPa}\left(4.1 \times 10^{6} \mathrm{psi}\right)$. Working backward, this corresponds to $\delta / P=0.000324 \mathrm{~cm} / \mathrm{N}$ $(0.000586 \mathrm{in} / \mathrm{lb})$.

The value of the effective radius of load application depends upon the degree of tilt of the cross section. The lower bound of $R_{e}$ is the inside radius, $R_{i}$; this corresponds to the case of the cross section being completely lifted off the loading bar except at the inside corner. If the load distribution is triangular with the maximum intensity being at the inside corner, $R_{e}=R_{i}+(b / 3)$. If the distribution is parabolic, $R_{e}+R_{i}+(2 / 5) b$.

Table 1 lists the values predicted for $G$ corresponding to the different cases.

The accepted value for the shear modulus of aluminum alloy is 25.9 to $26.2 \mathrm{GPa}$ (3.75 to 3.80 millions of psi). It is noted that the present data reduction with a loading distribution somewhere between a triangular and a parabolic distribution appears to reduce the error in prediction of the shear modulus.

Also, it is noted that inclusion of the effect of transverse shear deformation increases the predicted shear modulus by only about $1.5 \%$, which is not very significant.

\section{APPLICATION TO A COMPOSITE RING}

Greszczuk (1979) also presented data for a ring constructed of cross-ply-laminated T300/F178 graphite/epoxy. The data are as follows:

$$
\begin{gathered}
a=0.632 \mathrm{~cm}(0.249 \mathrm{in}) \\
b=0.262 \mathrm{~cm}(0.103 \mathrm{in}) \\
R=8.33 \mathrm{~cm}(3.28 \mathrm{in})
\end{gathered}
$$


Table 2. Predicted values of the shear modulus $\mathrm{G}_{\theta z}$ of graphite-epoxy ring.

\begin{tabular}{lc}
\hline Approach & $\mathrm{G}_{\mathbf{\theta z}}, \mathrm{GPa}$ (millions of psi) \\
\hline Equation (11) & $4.08(0.592)$ \\
Equation (11) without TSD & $4.07(0.590)$ \\
Greszczuk's equation & $4.17(0.604)$ \\
\hline
\end{tabular}

$$
E_{\theta}=80.83 \mathrm{GPa}\left(11.72 \times 10^{6} \mathrm{psi}\right)
$$

The derived parameters are as follows:

$$
\begin{gathered}
A=0.165 \mathrm{~cm}^{2}\left(0.0256 \mathrm{in}^{2}\right) \\
I=0.005515 \mathrm{~cm}^{4}\left(0.0001325 \mathrm{in}^{4}\right) \\
\beta=0.249 \text { for } a / b=2.42 \text { and } G_{r \theta} / G_{\theta z}=1 \\
J=0.00282 \mathrm{~cm}^{4}\left(0.0000677 \mathrm{in}^{4}\right) \\
\delta / P=0.0193 \mathrm{~cm} / \mathrm{N}(0.0338 \mathrm{in} / \mathrm{lb})
\end{gathered}
$$

$$
R_{e}=8.306 \mathrm{~cm} \text { (3.27 in), based on a parabolic load distribution }
$$

Table 2 shows the values predicted by the various methods. It is clear that for this material there are only small differences among the various equations.

It should be pointed out that the present analysis is strictly applicable only to macroscopically orthotropic specimens, e.g., circumferential wrap. However, it would probably be a good approximation in the case of many layers of a regular layup, e.g., cross-ply or angle-ply.

\section{CONCLUDING REMARKS}

An improved equation for predicting the torsional shear modulus from four-point ring-twist test data has been developed, validated by application to isotropic-material (6061 aluminum alloy) data, and also applied to high-strength graphite-epoxy data.

In the case of aluminum, the improvements in predicting shear modulus were significant, but in the case of graphite-epoxy, the improvement was not very significant. In both instances the effects of transverse shear deformation were negligible. 
Improved Equation for Shear Modulus Using Four-Point Ring-Twist Test Data

\section{REFERENCES}

Chamis, C.C. and Sinclair, J.H. 1977. "Ten-deg Off-axis Test for Shear Properties in Fiber Composites," Experimental Mechanics, 17:339-346.

Greszczuk, L.B. 1968. "Douglas Ring Test for Shear Modulus Determination of Isotropic and Composite Materials," Proceedings, 23rd Annual Technical and Management Conference, Society of the Plastics Industry, Washington, DC.

Greszczuk, L.B. 1979. "Four-point Ring Twist Test for Determining the Shear Modulus of Composites," Proceedings, 24th National Symposium, Society for the Advancement of Materials and Process Engineering, San Francisco, CA.

Greszczuk, L.B. 1981. "Application of Four-Point Ring-Twist Test for Determining Shear Modulus of Filamentary Composites," Test Methods and Design Allowables for Fibrous Composites, ASTM SPT 734, 21-33.

Jones, R.M. 1975. Mechanics of Composite Materials, Scripta Book Company, Washington, DC, p. 70.

Kintsis, T.Ya. and Shlitsa, R.P. 1978. "Determination of the Shear Modulus of Composites from Experiments on the Twisting of Circular Specimens," Polymer Mechanics, 14:764-767.

Lekhnitskii, S.G. 1981. Theory of Elasticity of an Anisotropic Body (translation of 1977 Russian edition), Mir Publishers, Moscow, pp. 283-286.

Penton, A.P. 1960. “A New Device for Determining Shear Properties of Reinforced Plastics,” Society of Plastics Engineers Journal, 16:1246-1247.

Pfafrod, G.O., Saulgozis, Yu.Zh., Knet-S, I.V., and Yanson, Kh.A. 1972. "Experimental Determination of the Shear Modulus of Compact Bone Tissue," Polymer Mechanics, 8:601-608.

Tarnopol'skii, Yu.M and Kincis, T. 1984. Static Test Methods for Composites, English translation, Van Nostrand Reinhold, New York, NY.

Whitney, J.M. 1967. "Experimental Determination of Shear Modulus of Laminated Fiber-Reinforced Composites," Experimental Mechanics, 7:447-448.

Whitney, J.M., Stansbarger, D.L., and Howell, H.B. 1971. "Analysis of the Rail Shear Test—Applications and Limitations," Journal of Composite Materials, 5:24-34. 